\title{
Spotted Fever in the Mediterranean
}

Rickettsia conorii, a member of the Spotted Fever Group (SFG) of rickettsiae, is the agent of the tickborne illness known as Mediterranean Spotted Fever (MSF). The organism was first described by Conor in Tunis in 1910 and has since been found to cause disease over a large geographical area encompassing not only the Mediterranean countries such as Italy, Spain, France and Israel but also parts of Africa, India and the Middle East.

The disease usually occurs following a tick bite, and Rhipicephalus sanguineus, the brown dog tick, is believed to be the main vector in Southern Europe. The dog (which may or may not be brown!) is the natural reservoir, often showing no signs of disease. The number of reported cases in man has risen in recent years ${ }^{1}$ and a survey in Salamanca, a rural province of Spain, showed a seropositivity rate of $73.5 \%$ in the general population for $R$. conorii. ${ }^{2}$ Positive serology was more common in those who reported close contact with the natural environment or with domestic animals. Climatic changes, possibly related to "global warming", have also been implicated as being at least partly responsible for this increased incidence. ${ }^{3}$ Tick replication and activity is enhanced by warm weather, explaining the peak incidence of the disease in the summer months. Closer contact with ticks, both by the expansion of cities into rural parts of endemic areas and by increased travel to Mediterranean countries, has caused a rise in the number of cases both in endemic and non-endemic areas.

The clinical picture can range from a mild, almost asymptomatic form to a severe illness (mortality rate up to $5 \%$ ), following an incubation period of 3-7 days. At the site of tick bite, most patients develop an eschar caused by focal necrosis of endothelial cells. Rickettsiae have a predilection for endothelial cells of small blood vessels which they invade and destroy. The eschar is pathognomonic of MSF but it is often found in secluded areas such as the axilla, behind the knee, between the buttocks or in other skin creasesticks tend to bite in hidden places! Frequently, this lesion goes unnoticed by both the patient, who on occasions presents with the tick still attached to the eschar, and by the clinician, unless a thorough examination is performed. Classically the patient develops the triad of fever, headache and rash. Conjunctivitis and meningism may also be present. The rash usually starts around the ankles and wrists, spreading centripetally to the rest of the body, frequently involving the palms and soles. It can be maculopapular or petechial and its "button-like" appearance is responsible for MSF also being called Boutonneuse fever. The skin lesions can range from very extensive to sparse, reflecting the degree of endothelial cell damage that also occurs in almost all deep organs.

In spite of the "typical" triad, MSF has to be distinguished from other infectious illnesses such as meningococcal septicaemia, infectious mononucleosis and measles. Haematological and biochemical investigations are unhelpful as there are no characteristic changes.

Isolation of the obligately intracellular bacteria is complicated by the hazard to laboratory staff and by their long generation times. Rickettsiae are classified as category III pathogens because they can be highly infectious in aerosols, so, although rickettsiae can be isolated by tissue culture, this is done only in a few reference laboratories with adequate containment facilities. Recently it has been reported that rickettsiae can be detected after as little as $48 \mathrm{~h}$ by centrifugation of the clinical sample on to tissue culture monolayers. ${ }^{4,5}$ Direct demonstration with immunofluorescent antibodies of rickettsiae from skin lesion biopsies is highly specific but lacks sensitivity.

Serological methods remain the commonest confirmatory tests for MSF. However, they provide only a retrospective diagnosis because the immunological response occurs late in the disease. The Weil-Felix reaction, the first serological assay to be developed for rickettsial diseases, is neither sensitive nor specific and is of historical interest only. At present an indirect immunofluorescent technique is the most widely used. The indirect haemagglutination test, which appears to be equally sensitive, is the only serological test available in the UK. This is performed at the PHLS centre for Applied Microbiology and Research, where approximately 40 cases of rickettsial diseases/year have been diagnosed in recent years. In the UK, many more cases are probably missed because of lack of awareness and failure to diagnose.

The polymerase chain reaction (PCR) with restriction fragment length polymorphism analysis is a useful epidemiological tool, allowing differentiation between different rickettsial species and between closely related isolates. ${ }^{6,7}$ However, its role in the clinical setting is not yet established; in the future it may provide a rapid diagnosis of rickettsial illnesses.

Prognosis of MSF is thought to be improved by early antibiotic therapy, thus a presumptive diagnosis has to be made on the basis of the history and clinical findings. This is difficult in non-endemic areas where 